\title{
The rational approach for controlling the spread of COVID-19 in Sri Lanka
}

\author{
Indika Karunathilake ${ }^{1}$, Rasnayaka Mudiyanse $^{1}$, Chamindri Witharana $^{2}$, Malik Peiris $^{3}$
}

Ceylon Medical Journal 2020; 65: 72-74

DOI: http://doi.org/10.4038/cmj.v65i3.9190

Sri Lanka is a upper-middle-income country with a population of 21.4 million and a per capita gross domestic product (GDP) of about US\$13,000.00 at purchasing power parity. Despite the total expenditure on health being relatively low (approximately $4.2 \%$ of the GDP), Sri Lanka is noted to have achieved health indicators that are comparable with high-income countires, with almost 100\% immunization coverage, life expectancy over 75 years, literacy rate over $90 \%$ and low mortality rates [1].

Sri Lanka's COVID-19 response is characterised by a strong focus on the preventive approach and contact tracing with rational utilization of available resources. The level of control achieved by this approach when applied within the Sri Lankan context is evident in the analyais of current situation. With only 134 cases per million population and a case fatality rate of $0.38 \%$, the Sri Lankan statistics are lower than the global average [2].
Globally as updated on 12th August 2020, there have been 20,525,986 confirmed cases of COVID-19, including 745,971 deaths, reported to WHO. In Sri Lanka, updated on $12^{\text {th }}$ August 2020, there have been 2,880 confirmed cases of COVID-19 with 11 deaths (WHO, 2020).

Even after 28 weeks since the detection of the first patient, most cases can still be traced to an identifiable origin. This indicates that the epidemic is contained within few clusters, without spilling into the community. Most of the early clusters and chains of transmission are no longer active.

The Sri Lankan response to COVID-19 needs to be understood within the context of health system of the country. Sri Lanka has a strong and well-established public health system with both preventive and curative healthcare provided free of charge by the Government health service [1].

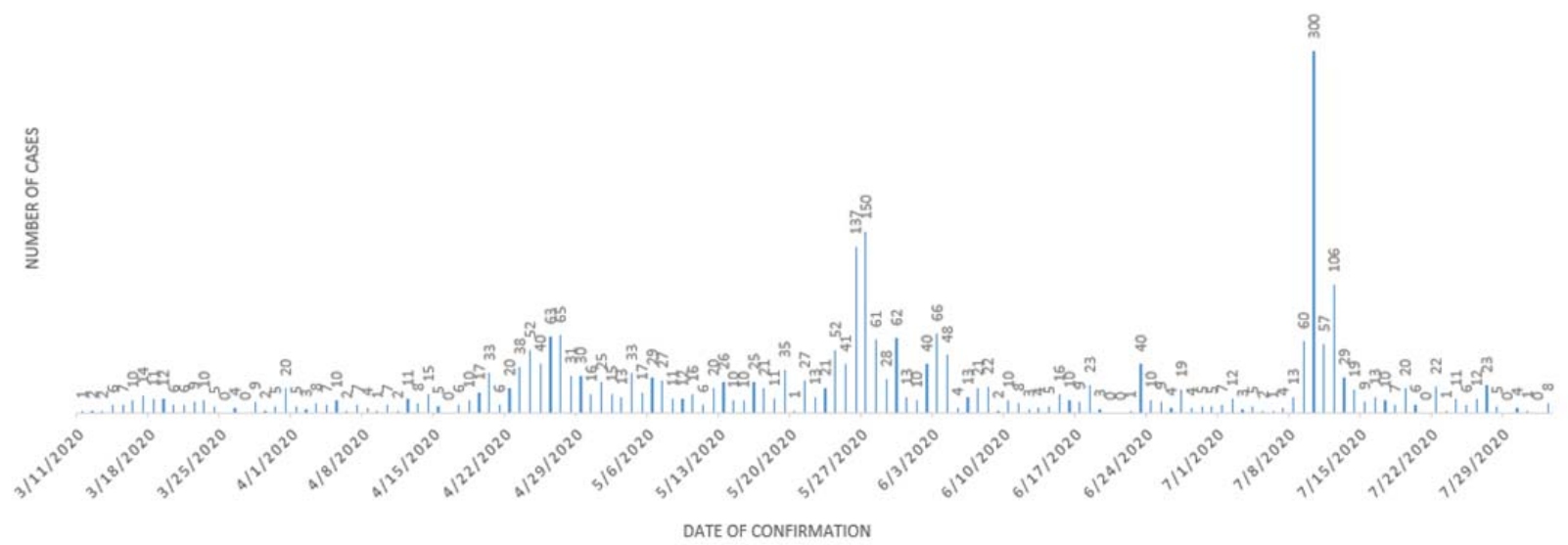

Figure 1. Epidemiology curve of COVID-19 patients, Sri Lanka (as of 30.07.2020).

${ }^{1}$ Sri Lanka Medical Association, ${ }^{2}$ University of Colombo, Sri Lanka, ${ }^{3}$ University of Hong Kong, Hong Kong.

Correspondence: IK, e-mail: <indika@medarc.cmb.ac.lk>. Received 30 June 2020 and revised version 13 August 2020 accepted 20 October 2020.

(i) This is an open-access article distributed under the terms of the Creative Commons Attribution License, which permits unrestricted use, distribution, and reproduction in any medium, provided the original author and source are credited. 
Sri Lanka's COVID-19 response is not very different from the prescribed measures practiced worldwide. However, the difference has been in the timing of those measures and the intensity with which they were applied. The national response was initiated prior to the detection of the first case. The Ministry of Health issued a preliminary social distancing advisory on $20^{\text {th }}$ January, a week before the first COVID-19 case was confirmed. This was followed by establishing screening at entry points to the country, mandatory institutional quarantine for travellers from high risk destinations, closure of educational institutions and work from home in order to enforce physical distancing [4].

Following a sudden influx of expatriate workers affected by the epidemic in Italy, the government announced a nation-wide curfew on $20^{\text {th }}$ March 2020. Everyone, except those working in the essential services, were required to stay home. Home delivery service of essential goods was established. These services were further streamlined by the creation of a special task force to distribute essential items [4].

A policy of preemptive quarantine and isolation of contacts even before they were tested for infection was followed. This approach reduced the margin of error, and the testing policy evolved based on this approach with quarantined individuals being tested prior to discharge.

The COVID-19 diagnostic test used in Sri Lanka is Reverse Transcriptase Polymerase Chain Reaction (RTPCR) test. The primary strength of molecular tests such as RT-PCR is that they directly detect COVID-19 in the early stages when the patient is infectious. The case definition for testing has been modified regularly based on the epidemiological data and the ground situation to allow flexibility in decision making while controlling for potential wastage of limited resources. Sri Lanka's policy of rational and methodical use of testing has been effective. From an average of $5 \%$ at the beginning of March, the test positivity rate had dropped to $1.73 \%$ by the time of writing $[1,2]$.

An extensive and systematic contact-tracing program was launched utilizing the public health system in collaboration with the national intelligence service and the security forces. The expertise, extensive network and stateof-the-art technology available with national intelligence service was maximally utilized. Geographic information systems (GIS) and Big-data analysis was used to identify the contacts of all three levels. The physical distancing of these contacts was implemented even before confirmation by RT-PCR testing by implementing supervised home quarantine, institutionalized quarantine, isolation of villages or a section of a locality [4].

Sri Lankan Security Forces, considering their organizational capacity, are given the major responsibility of establishing and managing quarantine centers. The efficiency of this system is evident by the fact that, by mid February 52 quarantine centers with the capacity to cater for over 5,000 people have been established. A negative RT-PCR is mandatory to be released from the quarantine camp [4].

All RT-PCR positive cases in Sri Lanka have been admitted to one of the five designated hospitals. They are subjected to two more RT-PCR tests prior to discharge to confirm that there is no viral shedding. At present, the caseload has not over-burdened the health care system. As of 12/08/2020 there is a cumulative number of 2880 confirmed cases, and out of them 978 cases are imported cases who have returned from other countries comprised of 946 Sri Lankans and 32 foreigners. Others are local cases and with two large identified clusters at the Naval Base in Welisara and the rehabilitation camp for substance abusers at Kandakadu. Over 2622 have fully recovered. Approximately 247 patients are currently hospitalized, of whom $90 \%$ are asymptomatic. Total number of deaths are 11 giving rise to a fatality rate of $0.39 \%$ [2].

The cluster of Navy officers formed 33\% from total infected cases. The imported cases from the other countries comprised with almost all with the Sri Lankan citizens gave rise to the $34 \%$ from all the confirmed infected patients whom were also controllable and identified [2].

Sri Lanka started RT-PCR testing at the international airport for overseas returnees and international arrivals. Depending on the results, they are being either diverted to the hospital management or quarantine centers [2].

However, the Sri Lankan approach is not without shortcomings and drawbacks. The flipside of the emphasis on COVID-19 reduced attention and disruption of routine medical services. Outpatient lists were cancelled and resulted in late diagnosis and death. Prevention and treatment services for noncommunicable diseases (NCDs) were severely disrupted since the COVID-19 pandemic began, specially diabetes and diabetes-related complications, cancer treatments and cardiovascular emergencies. Due to the cancellations of the planned treatments and a decrease in public transport available, a discontinuation and a decrease of health services occurred in the country. Sexual and Reproductive Health (SRH) services, both in the public and private sector, were heavily curtailed except for deliveries and pregnancy-related services.

Bringing the large clusters of Navy officers and later, inmates of Kandakadu rehabilitation center under control was a challenge. Sensationalized Media reports regarding the false positives lead to a confusion and affected the credibility of the process. The Kandakadu cluster with some who may be having links with illegal drug distribution networks, contact-tracing process has become more challenging. This factor also caries the risk of the epidemic moving into the community transmission stage. 
Since the end of May, Sri Lanka moved into the phase of gradually relaxing the strictly enforced restrictions, to recover from its economic downfall suffered in the hands of COVID-19. The strict adherence during the initial phase was followed by a loss of discipline, bringing the country to the verge of community transmission in six weeks. Gradually it was observed that key measures are not adequately applied and monitored, increasing the vulnerability of the country for a resurgence of COVID19. The disappearance of the healthy behaviors seems largely due to the fast relaxation of control measures indicating that the risk of COVID-19 is no more. This notion was extremely harmful.

During the recently concluded parliamentary elections, Sri Lanka Medical Association (SLMA) worked in close collaboration and supported National Election Commission of Sri Lanka for conducting the voting process in accordance with COVID-19 preventive guidelines.

However, frequent deviations and lack of adherence from prescribed precautionary measures were observed during the campaigns. The delay in the gazette of regulations to be followed hampered the power of health authorities to intervene.

After a period of four months, government schools were reopened on August 10. Repatriation process has also resumed after the conclusion of the national elections.

Above factors taken together, it is clear that the risk of community transmission or resurgence cannot be ruled out.

\section{Conclusion}

The key features of management of the COVID-19 pandemic in Sri Lanka are;

1. Early intervention by the government and Ministry of Health.

2. Extensive contact tracing.

3. Early isolation of all possible contacts pending confirmatory tests.

4. Free of charge, well-organized quarantine facilities for contacts with high degree of exposure.
5. Isolation of more vulnerable communities.

6. Hospitalization of all with positive test until RTPCR become negative.

7. Provision of free health care to all patients.

8. Proper awareness given to the public by the media with the leadership of the government health sector experts and military leaders.

The eradication of the COVID-19 may not be possible although containing the same through various measures is acheivable. As we deal with the day-to-day realities of a worldwide disease, there's a high possibility that the novel coronavirus could become endemic in the human population, much like influenza. If so, we could be living with COVID-19 for a long time. Therefore, we cannot predict when we would be returning back to our normal lifestyle, but need to rather adapt the 'new normal' lifestyle.

Sri Lanka's response to COVID-19 is rooted in a strong preventive approach and national level leadership. Sri Lanka is now approaching the phase of gradually relaxing the strictly enforced restrictions. While acknowledging the need for transition to normalcy and to initiate economic activities, it is of prime importance to remain focused on the primary objective of controlling the epidemic and without any set back in the gains achieved. A staggered and gradual strategy based of scientific principles needs to be adapted without compromising the present public health achievements.

\section{References}

1. World Health Organization. Sri Lanka Country Profile. Geneva: World Health Organization, 2020 (https://www. who.int/gho/countries/lka/country_profiles/en/).

2. Epidemiology Unit, Ministry of Health, Sri Lanka. Novel Coronavirus (2019-nCoV) - Situation Report - 141 ; 28/ 06/2020 - 10.00 am. https://www.epid.gov.lk/web/images/ pdf/corona_virus_report/sitrep-sl-en-28-06_10.pdf

3. World Health Organization. Novel coronavirus (2019nCoV): situation report - 160. https://www.who.int/docs/ default-source/coronaviruse/situation-reports/20200628covid-19-sitrep-160.pdf?sfvrsn=2fe1c658_2

4. Silva S. Sri Lanka's Approach in Combatting COVID-19: Country Presentation. APACPH-SLMA Virtual Conference on COVID-19, 2020-05-17. 